\title{
Equilibrium Modal Power Distribution Measurement of Step-index Hard Plastic Cladding and Graded-index Silica Multimode Fibers
}

Ruichen Tao ${ }^{1}$, Takehiro Hayashi², Manabu Kagami ${ }^{3}$, Shigeru Kobayashi ${ }^{4}$, Manabu Yasukawa², Hui Yang ${ }^{5}$, David Robinson ${ }^{5}$, Hadi Baghsiahi ${ }^{1}$, F. Aníbal Fernández¹, David R. Selviah ${ }^{1}$

${ }^{1}$ Department of Electronic and Electrical Engineering, University College London ${ }^{2} \mathrm{HAT}$ Lab. Inc.

${ }^{3}$ Toyota Central R \& D Labs, Inc., Information \& Communication Research Div. ${ }^{4}$ Tyco Electronics

${ }^{5}$ Arden Photonics Ltd

d.selviah@ucl.ac.uk, +44(0)20 76793056 


\section{Standard far field angular power distribution generation}

-1) Long multimode fibre so that the modal power distribution reaches it equilibrium distribution

- 2) Mode filter formed by wrapping an optical fibre around a mandrel sufficient times to reach an equilibrium distribution

- 3) A commercial device designed to generate a standard near field Encircled Flux, EF, distribution 


\section{Encircled Angular Flux Measurement Techniques}

- 1) The light from the source multimode fibre is allowed to diverge in free space onto a CCD camera chip placed in the far field.

Far field: $d>>2 D^{2} / \lambda, D$-fiber core diameter

-2) A commercial Fourier Transform or F-Theta lens system is used to convert the angular distribution into a spatial distribution which impinges onto a CCD camera chip. This is also referred to as the Far Field Pattern, FFP system. 


\section{Experimental arrangement}

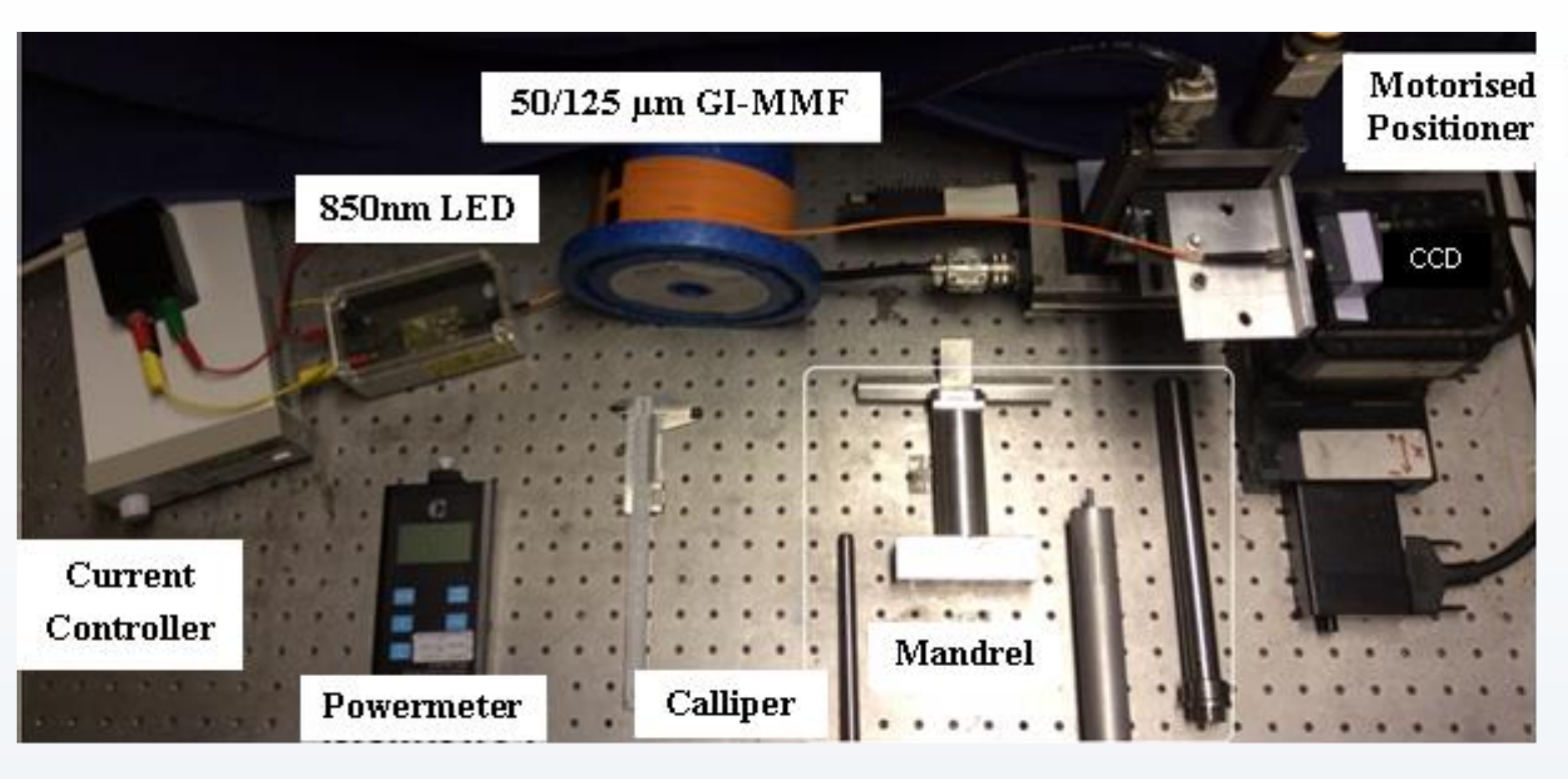

- LED1: HFE4050-014/FXA, controller: Thorlabs LDC202C

- LED2: Adamant FOLS-01

- GI-MMF: 50/125 $\mu \mathrm{m}, 20 \mathrm{~m}$

- HPCF: OFS CF01493-10

- Adapter: Thorlabs SM1FC

- CCD: Prosilica EC1600

- LED1/LED2: $850 \mathrm{~nm}$

- $50 \mu \mathrm{m}$ GI-MMF: Silica/Silica $N A=0.20 \pm 0.02, d=10 \pm 1 \mathrm{~mm}, \mathrm{a}_{\max } \sim 13^{\circ}$

- $200 \mu \mathrm{m}$ SI-MMF: HPCF $N A=0.37 \pm 0.02, d=105 \pm 1 \mathrm{~mm}, \mathrm{a}_{\max } \sim 26^{\circ}$ 


\section{Encircled Angular Flux}

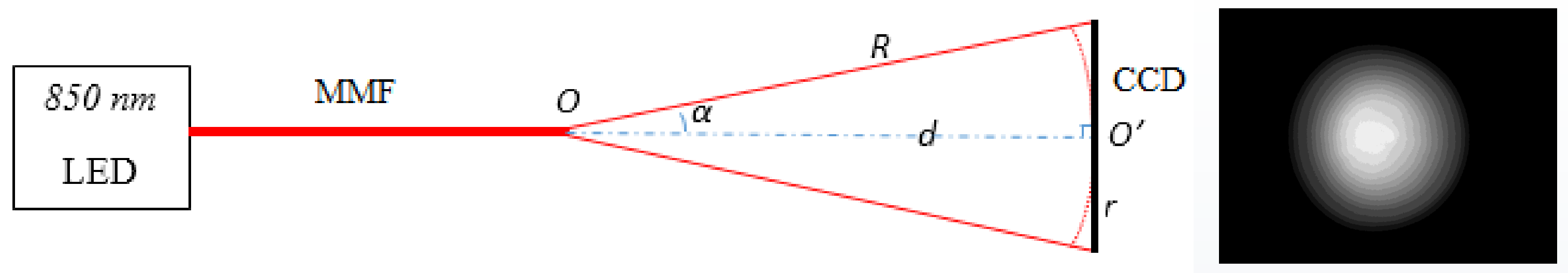

- Encircled angular flux (EAF): $\int P(\alpha) / P\left(\alpha_{\max }\right) \cdot d \alpha$

- $\tan (\alpha)=r / d$

- $\alpha_{\max }>\arcsin (\mathrm{NA})$

- $\Delta \alpha$ : related to pixel size

- O': centroid of far field image 


\section{EAF: Free space divergence onto CCD measurement method}

- Labelling convention example:

- $\mathrm{J}(\mathrm{min})-1533-10 \mathrm{~mm}-52 \mathrm{~ms}$

Label

- $J(\min )$ : LED2 at minimum output

- 1533: "modcon" serial No., Modcon 1

- M250471: "modcon" serial No., Modcon 2

- $x \times$ ms: exposure time of CCD

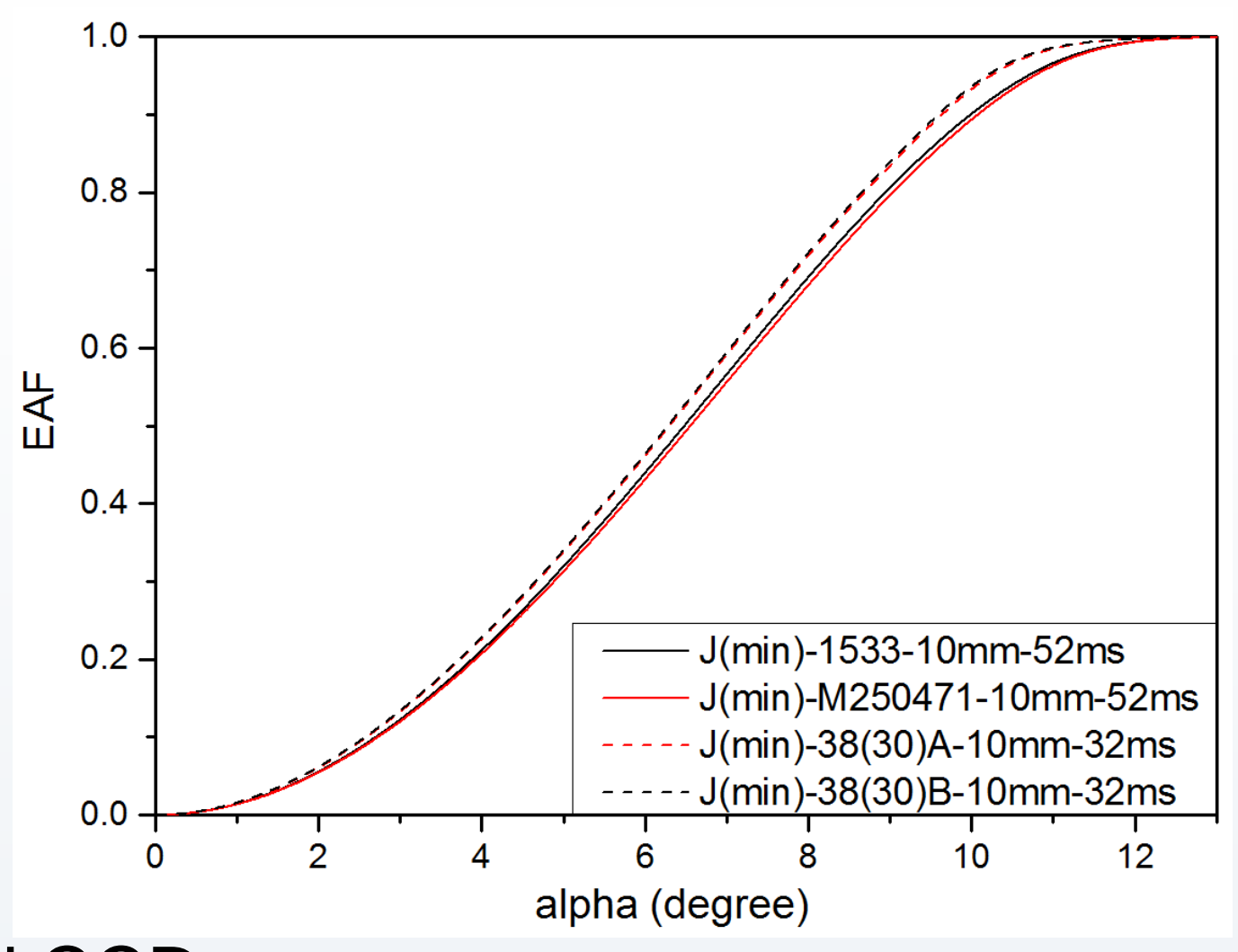

- $10 \mathrm{~mm}$ : the distance between fiber end and CCD

- 38(30): $38 \mathrm{~mm}$ diameter Mandrel with 30 turns of $50 \mu \mathrm{m}$ GI-MMF

- $A / B$ : side $A$ or side $B$ of the fiber under test connected to the LED 


\section{EAF: F- $\theta$ Lens measurement method}
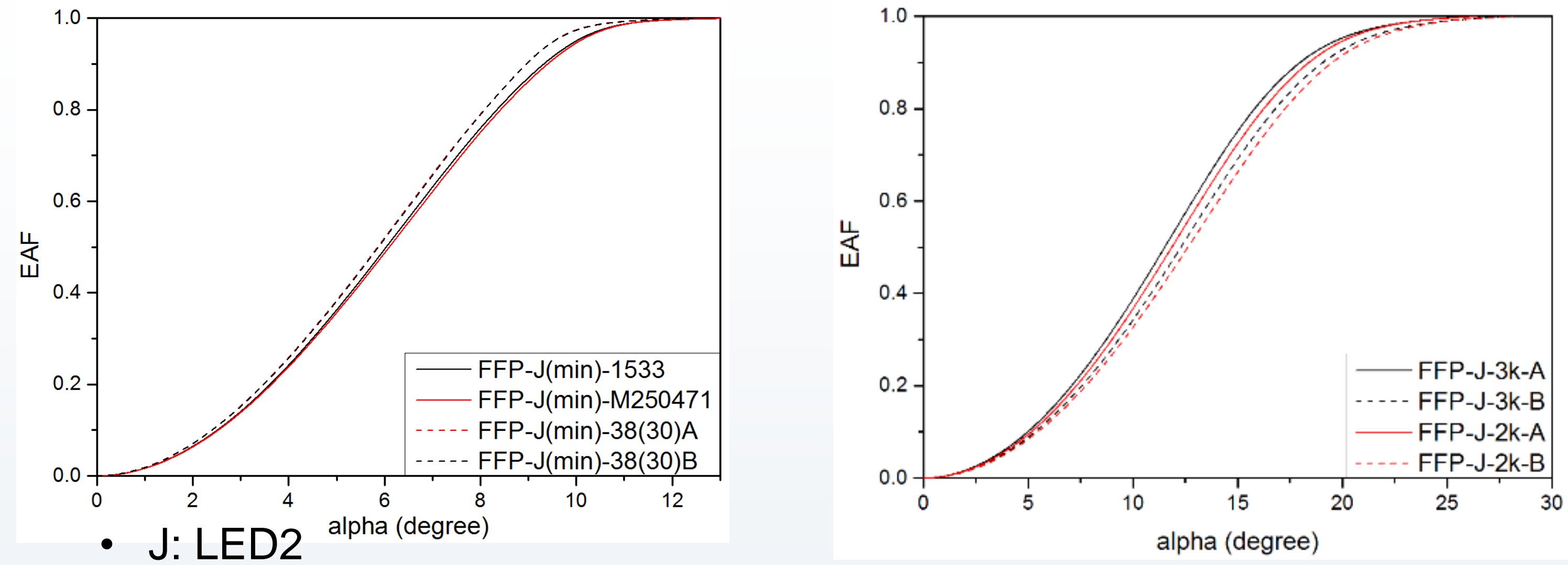

- 1533: "modcon" serial No. ; M250471: "modcon" serial No.

- 38(30): $38 \mathrm{~mm}$ diameter Mandrel with 30 turns of $50 \mu \mathrm{m}$ GI-MMF

- $A / B$ : side $A$ or side $B$ of the fiber under test connected to the LED

- $2 / 3 \mathrm{k}: 2 \mathrm{~km}$ or $3 \mathrm{~km} \mathrm{HPCF}$ 
EAF: Comparison of free space divergence and F-Theta lens measurement methods

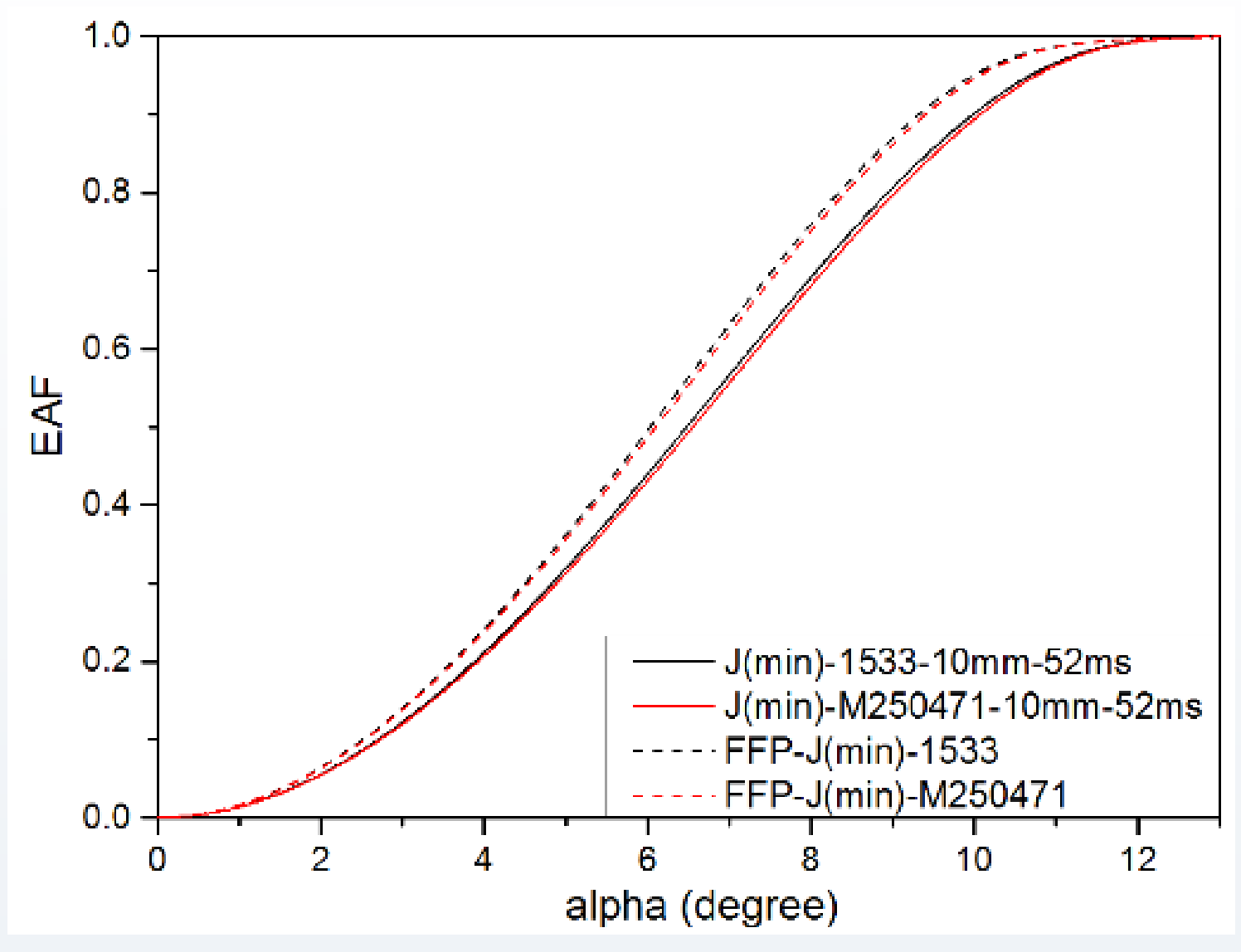

- (1)“Modcon"

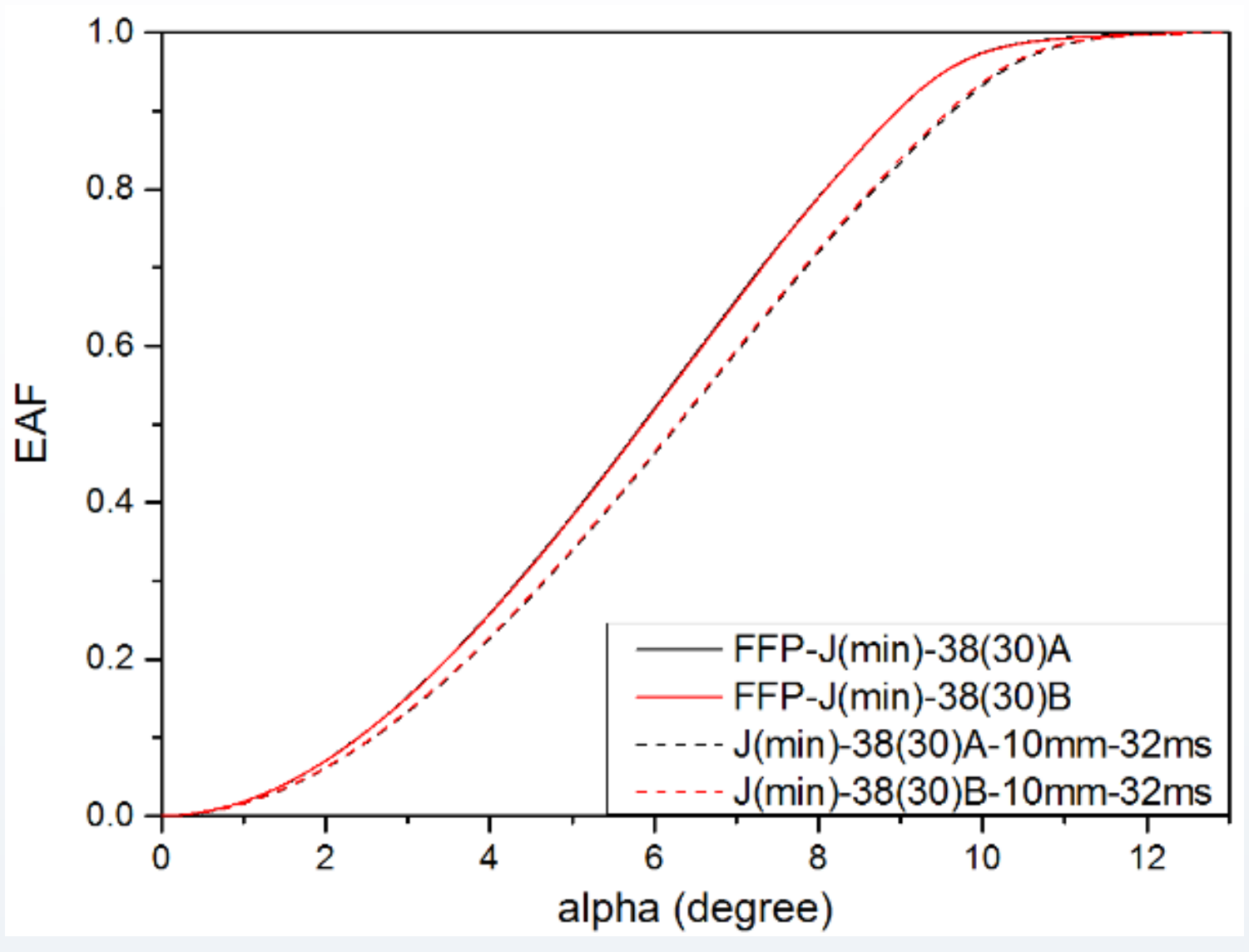

- (2) Mandrel: 30 turns 


\section{EAF: compare three EMD methods}

- "Modcon": 1533, M250471

- Mandrel: $38 \mathrm{~mm}$ diameter, wrapped with $50 \mu \mathrm{m}$ GI-MMF

- Long fiber: 2 km, 3 km HPCF
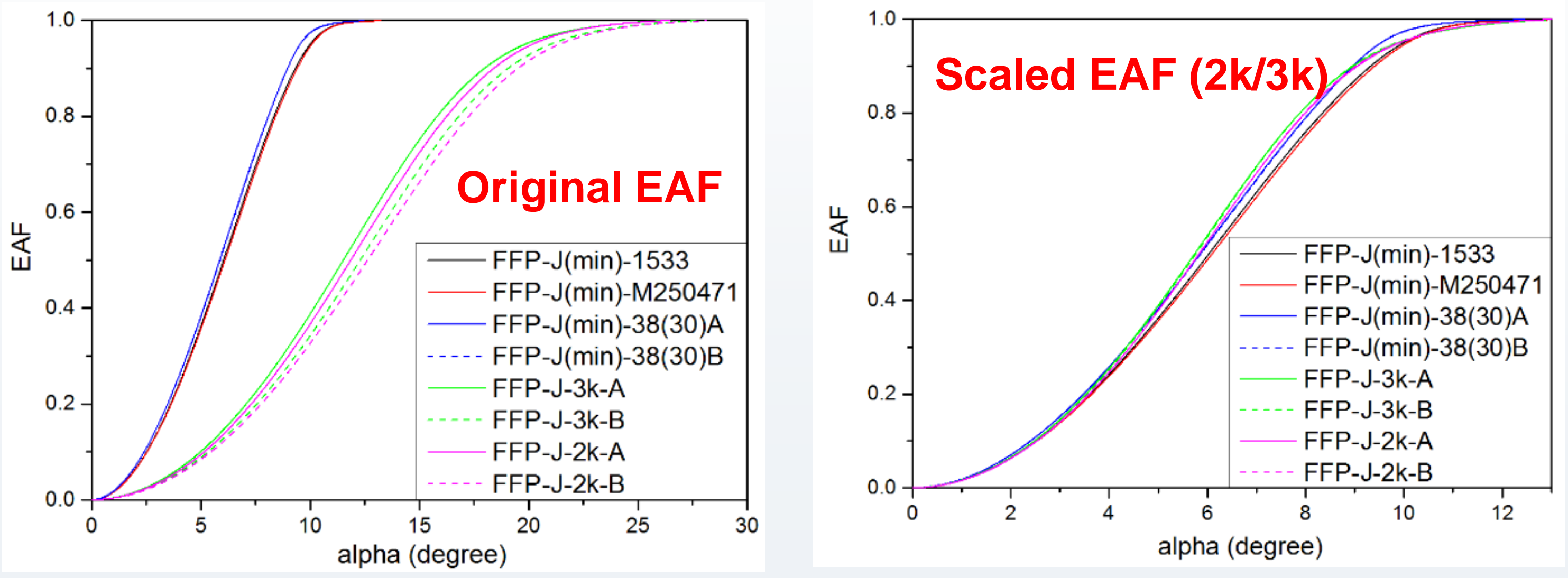


\section{Conclusions}

- 30 turns of a GI-MMF around a $38 \mathrm{~mm}$ diameter mandrel were found to be sufficient to reach an equilibrium mode distribution.

- The two different near field Encircled Flux "modcon" sources gave very similar EAFs to each other and to the wrapped mandrel source

- The free space divergence measurement method gave similar but not exactly the same results for EAF as the F- $\theta$ lens method. Some further research is needed to bring them into closer agreement.

- At least $3 \mathrm{~km}$ of HPCF is required to reach an equilibrium mode distribution

- A new method of scaling the angle by the maximum angle brings all of the curves very close together so that a single standard EAF curve can be defined as long as the scaling factor is stated. 


\section{Acknowledgements}

TOYOTA CENTRAL R\&D LABS., INC.

ADNTMN Synos
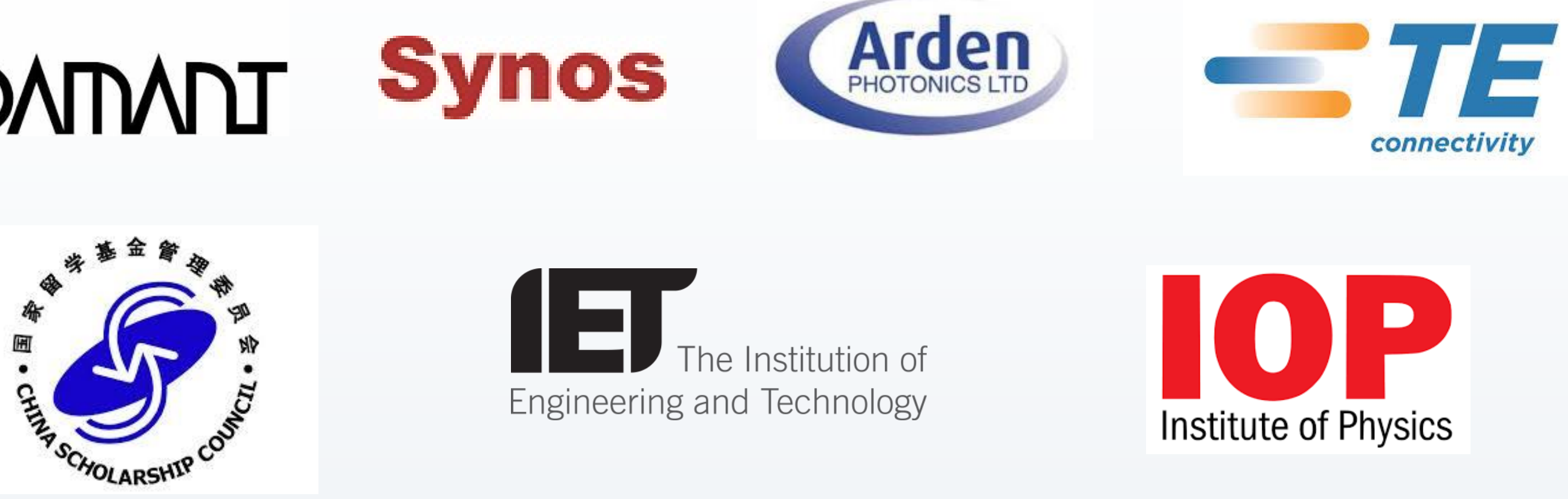

- Synergy Optosysterms Co., Ltd Booth Number: 4807

- Adamant Co., Ltd. Booth Number: 6006

- Arden Photonics Ltd Botth Number: 5515 